doi:10.13108/2021-13-4-41

\title{
CONVERGENCE RATE OF ONE CLASS OF DIFFERENTIATING SUMS
}

\author{
M.A. KOMAROV
}

\begin{abstract}
We consider a differentiation formula for functions analytic in the circle $|z|<1$ : $a z f^{\prime}(z)=n f(0)-\sum_{k=1}^{n} f\left(\lambda_{k} z\right)+R_{n}(z)$. Here $a \neq 0$ is a real constant, $n=1,2, \ldots$, while complex parameters $\lambda_{k}=\lambda_{n, k}(a), k=1, \ldots, n$, are defined as the unique solution of a discrete moment system for Newtonian power sums $\lambda_{1}^{m}+\cdots+\lambda_{n}^{m}=-m a, m=$ $1, \ldots, n$. Under such choice of the parameters, the function $R_{n}(z)=R_{n}(a, f ; z)$, which is the remainder in the formula, is of order $O\left(z^{n+1}\right)$ as $z \rightarrow 0$. In this work we show that for each fixed $a>0$ and each $n \geqslant 3 \alpha(\alpha:=\max \{a ; 1\})$ the domain of the applicability of the formula contains the circle $|z|<\exp (-3 \sqrt{v}-2 v), v:=\alpha /(n+1)$, the radius of which tends to one as $n \rightarrow \infty$. We establish an exponential convergence rate of differentiating sums to $n f(0)-a z f^{\prime}(z)$ in the same circle. This result completes and extends essentially previous results by V.I. Danchenko (2008) and P.V. Chunaev (2020), which, respectively for the cases $a=-1$ and $-n \leqslant a<0$ established the convergence of the differentiating formula but only in the domains contained in fixed compact subsets of the unit circle. The proof of the main results of the paper is based essentially on an approach for constructing a solution for the mentioned moment system; this approach differs essentially from that by Danchenko and Chunaev.
\end{abstract}

Keywords: differentiation of analytic functions, differentiating sums, $h$-sums, convergence rate.

Mathematics Subject Classification: 30E10, 41A25, 65D25

\section{INTRODUCTION}

Let

$$
h(z)=\sum_{m=0}^{\infty} h_{m} z^{m}
$$

be a function analytic in the unit disk $D=\{z:|z|<1\}$. V.I. Danchenko [1, Sect. 2.4] proposed a formula for a numerical differentiation

$$
(z h(z))^{\prime}=\sum_{k=1}^{n} \mu_{k} h\left(\mu_{k} z\right)+\varepsilon_{n}(z), \quad \mu_{k}=\mu_{n, k} \quad(n=1,2, \ldots),
$$

where complex numers $\mu_{1}, \ldots, \mu_{n}$ are uniquely determined by the condition

$$
\varepsilon_{n}(z)=O\left(z^{n}\right) \quad(z \rightarrow 0) .
$$

Namely, since

$$
(z h(z))^{\prime}-\sum_{k=1}^{n} \mu_{k} h\left(\mu_{k} z\right)=\sum_{m=1}^{\infty}\left(m-\sum_{k=1}^{n} \mu_{k}^{m}\right) h_{m-1} z^{m-1},
$$

then $\mu_{1}, \ldots, \mu_{n}$ is a unique solution of system

$$
\mu_{1}^{m}+\cdots+\mu_{n}^{m}=m, \quad m=1, \ldots, n,
$$

M.A. Komarov, Convergence rate of one class of Differentiating sums.

(C) Komarov M.A. 2021.

Submitted September 2, 2020. 
for Newtonian power sums. One of the ways of solving the system is based on applying Newton recurrent formulae, see [1] for more details. It is important to note that the quantities $\mu_{k}$ are independent of the function $h$. The sums of form $\sum_{k=1}^{n} \mu_{k} h\left(\mu_{k} z\right)$ are called $h$-sums.

Each analytic in $D$ function

$$
f(z)=\sum_{m=0}^{\infty} f_{m} z^{m}
$$

can be represented as $f(z)=f(0)+z h(z)$ with a certain analytic in $D$ function $h$ and this is why formula (1.1) is equivalent to the formula

$$
z f^{\prime}(z)=-n f(0)+\sum_{k=1}^{n} f\left(\mu_{k} z\right)+\varrho_{n}(z), \quad \varrho_{n}(z)=O\left(z^{n+1}\right) .
$$

Recently P.V. Chunaev considered a generalization of formula (1.2) [2, Sect. 3.3.5]:

$$
z f^{\prime}(z)=-t f(0)+\frac{t}{n} \sum_{k=1}^{n} f\left(\tilde{\mu}_{k} z\right)+\tilde{\varrho}_{n}(z), \quad \tilde{\varrho}_{n}(z)=O\left(z^{n+1}\right),
$$

by introducing an additional real parameter $t \neq 0$. It is clear that $(1.3)$ coincides with 1.2$)$ for $t=n$ and that both formulae are exact on polynomials of degree at most $n$. The quantities $\tilde{\mu}_{k}=\tilde{\mu}_{n, k}(t)$ are determined by the system

$$
\tilde{\mu}_{1}^{m}+\cdots+\tilde{\mu}_{n}^{m}=\frac{m n}{t}, \quad m=1, \ldots, n .
$$

In works [1], 2], the domains of applicability of differentiation formulae (1.2), (1.3) were established and the errors were estimated. For instance, it was shown in [2] that if

$$
\left|f_{m}\right| \leqslant 1, \quad m=0,1,2, \ldots,
$$

then for all $t \geqslant 1$

$$
\left|\tilde{\varrho}_{n}(z)\right| \leqslant \frac{2 \sqrt[n]{t}|(2 n+1) z|^{n+1}}{(\sqrt[n]{t}-(2 n+1)|z|)^{2}}, \quad|z|<\frac{\sqrt[n]{t}}{2 n+1} .
$$

A simple analysis of the proof shows that

a) this estimate holds true also for $t \leqslant-1$ if we replace $t$ by $|t|$ in it,

b) as $|t| \asymp n$ we can state more, for instance, as $t=n$, by Theorem 4(b) in [2] with $r_{0}=n$, $\gamma=a=1$ we obtain an estimate for error in formula $(1.2)$ :

$$
\left|\varrho_{n}(z)\right| \leqslant 2 n \frac{|3 z|^{n+1}}{(1-3|z|)^{2}}, \quad|z|<\frac{1}{3}
$$

Nevertheless, the domains of applicability of formulae $(1.2),(1.3)$ found in [1], 2] are essentially less than the analyticity domain of the function $f$, which contains the unit circle. In the present work, for each fixed $t<0$, we construct a new estimate for the error in formula (1.3), which can be applied in a circle of form $|z|<r$, where $r=r(t, n) \rightarrow 1$ as $n \rightarrow \infty$. The way of the proof differs substantially from the approaches in works [1], [2] and it can not be generalized for the case $t>0$.

Other effective modifications of the method of differentiating $h$-sums different from (1.3) were constructed by the author [3] and by V.I. Danchenko and P.V. Chunaev [4]. In the first of these works the differentiating differences of $h$-sums was studied, while in the other the differentiation was by amplitude-frequency sums $\sum_{k=1}^{n} \lambda_{k} h\left(\mu_{k} z\right)$. In [5] A.V. Fryantsev applied the method of $h$-sums for approximating differential polynomials of certain form generalizing the differentiation operator $(z h(z))^{\prime}$. 


\section{RESUlts}

Letting $a=-n / t$, for $t<0$ we write formula $(1.3)$ as

$$
a z f^{\prime}(z)=n f(0)-\sum_{k=1}^{n} f\left(\lambda_{k} z\right)+R_{n}(z), \quad a>0, \quad \lambda_{k}=\lambda_{n, k}(a),
$$

where

$$
R_{n}(z)=R_{n}(a, f ; z)=O\left(z^{n+1}\right), \quad z \rightarrow 0, \quad \lambda_{n, k}(a):=\tilde{\mu}_{n, k}\left(-\frac{n}{a}\right) .
$$

It is clear that $\lambda_{1}, \ldots, \lambda_{n}$ is a unique solution of the system

$$
\lambda_{1}^{m}+\cdots+\lambda_{n}^{m}=-m a, \quad m=1, \ldots, n,
$$

while the error term in formula 2.1 reads as

$$
R_{n}(a, f ; z)=\sum_{m=n+1}^{\infty}\left(m a+S_{m}\right) f_{m} z^{m}, \quad S_{m}=S_{n, m}(a):=\sum_{k=1}^{n} \lambda_{k}^{m} .
$$

Theorem 2.1. Let $a>0, n \geqslant 3 \alpha$ and $r=r_{n}(\alpha)$, where

$$
r_{n}(\alpha)=\exp \left(-\frac{3 \sqrt{\alpha}}{\sqrt{n+1}}-\frac{\alpha+1}{n+1}\right), \quad \alpha=\max \{a ; 1\} .
$$

Then the solution $\lambda_{1}, \ldots, \lambda_{n}$ of system (2.2) obeys the estimate

$$
\Lambda_{n}(a):=\max _{1 \leqslant k \leqslant n}\left|\lambda_{k}\right|<r^{-1} .
$$

Theorem 2.1 will be proved in Section 3. We recall that according [2], the parameters in formula (1.3) satisfy the estimate

$$
\max _{1 \leqslant k \leqslant n}\left|\tilde{\mu}_{n, k}(t)\right| \leqslant \frac{2 n+1}{\sqrt[n]{|t|}}, \quad t \neq 0,
$$

which for $t:=-n / a$ yields the following estimate for the quantities $\Lambda_{n}(a)$ :

$$
\Lambda_{n}(a) \leqslant \frac{2 n+1}{\sqrt[n]{n}} \sqrt[n]{|a|}
$$

We see that for each fixed $a>0$ estimate (2.3) is sharper in order of $n$ since as $n \rightarrow \infty$, an asymptotics holds:

$$
r^{-1}=\left(r_{n}(\alpha)\right)^{-1} \sim 1+\frac{3 \sqrt{\alpha}}{\sqrt{n}}<1+\frac{3 \sqrt{a+1}}{\sqrt{n}} .
$$

Let us find the domain of applicability and an estimate for the remainder in formula (2.1).

Theorem 2.2. Let $a>0, n \geqslant 3 \alpha$ and $r=r_{n}(\alpha)$, where $\alpha=\max \{a ; 1\}$. Let $\left|f_{m}\right| \leqslant 1$ for all $m \geqslant n+1$. Then

$$
\left|R_{n}(a, f ; r z)\right| \leqslant(n+1) \frac{|z|^{n+1}}{1-|z|}, \quad|z|<1 .
$$

Proof. In view of 2.3$)$ we have $\left|S_{m}\right|<n r^{-m}(m \geqslant n+1)$ and

$$
\left|R_{n}(a, f ; r z)\right| \leqslant \sum_{m=n+1}^{\infty}\left(m \alpha r^{m}+n\right)|z|^{m}, \quad m \alpha<e^{3 \sqrt{m \alpha}} \leqslant e^{\frac{3 m \sqrt{\alpha}}{\sqrt{n+1}}}<r^{-m},
$$

and this completes the proof. 
Remark 2.1. For each $n \geqslant 3$, the maximal value of $a$, under which the assumptions of Theorem 2.1 are satisfied is equal to $n / 3$. According to (2.3), the corresponding value of quantity $\Lambda_{n}$ do not exceed

$$
\exp \left(\frac{3 \sqrt{3}+1}{3}\right)+o(1) \approx 7.89, \quad n \rightarrow \infty .
$$

However, it is easy to establish a sharper bound, see Remark 4.1 in Section 4.1:

$$
\Lambda_{n}\left(\frac{n}{3}\right)<\exp \left(\frac{2 \sqrt{3}+1}{3}\right)=4.42838 \ldots \quad(n \geqslant 50) .
$$

Corollary 2.1. If a function $f$ is analytic in $D$ and

$$
|f(z)| \leqslant 1, \quad|z|<1,
$$

then for $r=(4.4284)^{-1}=0.2258 \ldots$ the estimate

$$
\left|\frac{z f^{\prime}(z)}{3}-f(0)\right| \leqslant 1, \quad|z| \leqslant r
$$

holds true.

Proof. Formula (2.1) is exact for polynomials $P$ of degree at most $n$, in particular, for $a=n / 3$ we have an identity

$$
n \frac{z P^{\prime}(z)}{3} \equiv n P(0)-\sum_{k=1}^{n} P\left(\lambda_{k}^{*} z\right), \quad \lambda_{k}^{*}=\lambda_{n, k}\left(\frac{n}{3}\right) .
$$

As $n \geqslant 50$ we let $f(z)=P_{n}(z)+F_{n}(z)$, where $P_{n}$ is the $n$th Maclaurin polynomial of the function $f, P_{n}(0)=f(0)$. By the identity and estimate $(2.4)$, in the disk $|z| \leqslant r$ we have

$$
\begin{aligned}
& \left|\frac{z P^{\prime}(z)}{3}-f(0)\right|=\frac{1}{n}\left|\sum_{k=1}^{n} P_{n}\left(\lambda_{k}^{*} z\right)\right| \leqslant \max _{|z|=\delta}\left|P_{n}(z)\right|, \\
& \delta:=r \cdot \Lambda_{n}\left(\frac{n}{3}\right)<(4.4284)^{-1} \cdot 4.42839<1 .
\end{aligned}
$$

Therefore,

where

$$
\left|\frac{z f^{\prime}(z)}{3}-f(0)\right| \leqslant 1+\Delta_{n}, \quad|z| \leqslant r
$$

$$
\Delta_{n}:=\max _{|z|=\delta}\left|F_{n}(z)\right|+\max _{|z|=r}\left|\frac{z}{3} F_{n}^{\prime}(z)\right| \rightarrow 0 \quad \text { as } \quad n \rightarrow \infty
$$

due to the boundedness of the function $f$. Since $n$ is arbitrary, we arrive at the needed statement. The proof is complete.

The identity in the estimate in Corollary 2.1 is attained at a constant function $f(z) \equiv 1$. A maximal possible value of the radius $r$ in this estimate can be obtained from a general theorem by G.M. Goluzin [6] and it is equal to

$$
r_{\max }=\frac{4-\sqrt{7}}{3}=0.4514 \ldots
$$

Indeed, if we denote the coefficients of the function $f$ by $f_{0}, f_{1}, \ldots$, then

$$
\frac{z f^{\prime}(z)}{3}-f(0)=\sum_{m=0}^{\infty} \gamma_{m} f_{m}, \quad \gamma_{0}:=-1, \quad \gamma_{m}:=\frac{m z^{m}}{3} \quad(m \geqslant 1) .
$$

Thus, inequality

$$
\left|\frac{z f^{\prime}(z)}{3}-f(0)\right| \leqslant 1, \quad|z| \leqslant r
$$


is equivalent to

$$
\left|\sum_{m=0}^{\infty} \gamma_{m} f_{m}\right| \leqslant\left|\gamma_{0}\right|=1, \quad \gamma_{m}=\frac{m z^{m}}{3}, \quad|z|=r .
$$

But according to Theorem 1 and formula (18) in [6], inequality (2.5) holds for all functions in the class mentioned in Corollary 2.1 if and only if

$$
\left|\sum_{m=0}^{\infty} \gamma_{m} \zeta^{m}\right| \geqslant\left|\sum_{m=1}^{\infty} \gamma_{m} \zeta^{m}\right| \quad \text { on } \quad|\zeta|=1
$$

Summing up the series, we obtain:

$$
\left|-1+\frac{w}{3(1-w)^{2}}\right| \geqslant\left|\frac{w}{3(1-w)^{2}}\right| \quad(w:=z \zeta, \quad|w|=r),
$$

which is equivalent to the condition

$$
\left|(w-1)^{2}-\frac{w}{3}\right| \geqslant r \quad(|w|=r) .
$$

The minimum of the left hand side in this inequality on the circumference $|w|=r<1$ is obviously attained as $w:=|w|=r$ and is equal to $\left|(r-1)^{2}-r / 3\right|=: h(r)$. If $r \in\left(r_{1}, 1\right)$, where

$$
r_{1}:=\frac{7-\sqrt{13}}{6} \approx 0.5657
$$

then $h(r) \leqslant 13 / 35<r_{1}$ and inequality $h(r) \geqslant r$ hence fails. If $r \in\left(0, r_{1}\right)$, then the expression under the absolute value sign is positive and the inequality $h(r) \geqslant r$ becomes

$$
(r-1)^{2}-\frac{r}{3} \geqslant r
$$

Choosing a maximal among its solutions lying in an admissible interval $\left(0, r_{1}\right)$, we arrive at the sought $r_{\max }$.

\section{Proof of Theorem 2.1}

3.1. Solution of system $(2.2)$. We consider the function

$$
g(z)=\exp \frac{a(1+z)}{2(1-z)}, \quad|z|<1, \quad a \in \mathbb{C}, \quad a \neq 0,
$$

and its Maclaurin series [7, Sect. 11.2]

$$
g(z)=\sum_{n=0}^{\infty} c_{n} z^{n}, \quad c_{n}=c_{n}(a)=e^{\frac{a}{2}} \sum_{k=1}^{n}\left(\begin{array}{c}
n-1 \\
k-1
\end{array}\right) \frac{a^{k}}{k !} .
$$

The roots of the $n$th Maclaurin polynomial

$$
g_{n}(z)=\sum_{k=0}^{n} c_{k} z^{k}, \quad g_{n}(0)=e^{\frac{a}{2}}
$$

are non-zero; we denote them by $z_{k}=z_{n, k}(a), k=1, \ldots, n$. It is obvious that

$$
\frac{g^{\prime}(z)}{g(z)}-\frac{g_{n}^{\prime}(z)}{g_{n}(z)}=O\left(z^{n}\right) \quad(z \rightarrow 0) .
$$

On the other hand, in some neighbourhood of the point $z=0$ we have:

$$
\frac{g^{\prime}(z)}{g(z)}=\frac{a}{(1-z)^{2}}=a \sum_{m=1}^{\infty} m z^{m-1}
$$


and

$$
\frac{g_{n}^{\prime}(z)}{g_{n}(z)}=\sum_{k=1}^{n} \frac{1}{z-z_{k}}=-\sum_{m=1}^{\infty}\left(\sum_{k=1}^{n} z_{k}^{-m}\right) z^{m-1} .
$$

Therefore, for all $n=1,2, \ldots$ and each complex $a \neq 0$ we have

$$
z_{1}^{-m}+\cdots+z_{n}^{-m}=-m a, \quad m=1, \ldots, n .
$$

Hence, a unique solution of system 2.2 reads as

$$
\lambda_{n, k}(a):=\left(z_{n, k}(a)\right)^{-1}, \quad k=1, \ldots, n .
$$

3.2. Estimate $(2.3)$ in case $a \geqslant 1$. It is known [7, Sect. 11.2] that $c_{n}(a)=e^{2 \sqrt{a n}(1+o(1))}$ as $n \rightarrow \infty(a>0)$. We shall need an explicit estimate for quantities $c_{n}(a)$ from above and one elementary inequality.

Lemma 3.1. As $a \geqslant 1, n \geqslant 3 a$, we have

$$
c_{n}(a)<\frac{1}{4} e^{\left(\frac{5}{2}-\sqrt{3}\right)(a+1)} e^{\frac{3}{2}} e^{2 \sqrt{a n}}<e^{a+1} e^{2 \sqrt{a n}} .
$$

Lemma 3.2. As $b \geqslant 1, x>1$, we have $e^{-b x}+e^{-\frac{b}{x}}<1$.

The proofs of these two lemmata will be provided in Section 4 .

By means of these lemmata, as $n \geqslant 3 a \geqslant 3$, we establish the estimate

$$
\left|g_{n}(z)\right|>0, \quad|z| \leqslant r_{n}(a)=e^{x_{n}}, \quad x_{n}:=-\frac{3 \sqrt{a}}{\sqrt{n+1}}-\frac{a+1}{n+1},
$$

which in view of $(3.1)$ implies immediately 2.3$)$ for the case $a \geqslant 1$.

Indeed, $|g(z)|>1$ in the circle $D$ since

$$
\operatorname{Re} \frac{a(1+z)}{2(1-z)}=\frac{a}{2} \frac{1-|z|^{2}}{1-2 \operatorname{Re} z+|z|^{2}}>0 .
$$

In view of Lemma 3.1 , in the circle $|z| \leqslant e^{x_{n}}$ we have:

$$
\left|g_{n}(z)\right| \geqslant|g(z)|-\sum_{k=n+1}^{\infty} c_{k}|z|^{k}>1-T_{n}(a), \quad T_{n}(a):=\sum_{k=n+1}^{\infty} e^{a+1} e^{2 \sqrt{a k}} e^{k x_{n}} .
$$

We are going to show that $T_{n}(a)<1$. As $k \geqslant n+1$ we have

$$
a+1+2 \sqrt{a k}+k x_{n} \leqslant 2 \sqrt{a k}-\frac{3 \sqrt{a}}{\sqrt{n+1}} k \leqslant-\frac{\sqrt{a}}{\sqrt{n+1}} k,
$$

and this is why

$$
T_{n}(a)<\sum_{k=n+1}^{\infty}\left(e^{-\frac{\sqrt{a}}{\sqrt{n+1}}}\right)^{k}=\frac{e^{-\sqrt{a(n+1)}}}{1-e^{-\frac{\sqrt{a}}{\sqrt{n+1}}}} .
$$

It remains to note that $e^{-\sqrt{a(n+1)}}<1-e^{-\frac{\sqrt{a}}{\sqrt{n+1}}}$ by Lemma 3.2 .

Remark 3.1. The positivity of the parameter $a$ is essential since in the case $a<0$ the absolute value $|g(z)|$ is not separated from zero in $D$ :

$$
g(x) \sim e^{\frac{a}{1-x}} \rightarrow 0 \quad(x>0, x \rightarrow 1-0) .
$$


3.3. Estimate $(2.3)$ in case $0<a<1$. In view of $(3.1)$ and $\alpha=1$ it is sufficient to establish that $\left|g_{n}(z)\right|>0$ in the circle $|z| \leqslant r_{n}(1), n \geqslant 3$. But as $a \in(0,1)$ we have

$$
c_{n}(a) e^{-\frac{a}{2}}=\sum_{k=1}^{n}\left(\begin{array}{l}
n-1 \\
k-1
\end{array}\right) \frac{a^{k}}{k !}<a \sum_{k=1}^{n}\left(\begin{array}{l}
n-1 \\
k-1
\end{array}\right) \frac{1}{k !}=a e^{-\frac{1}{2}} c_{n}(1), \quad n \geqslant 1,
$$

which yields

$$
c_{n}(a)<a e^{\frac{a-1}{2}} c_{n}(1)<c_{n}(1)
$$

Therefore,

$$
\sum_{k=n+1}^{\infty} c_{k}(a)\left(r_{n}(1)\right)^{k}<\sum_{k=n+1}^{\infty} c_{k}(1)\left(r_{n}(1)\right)^{k}<T_{n}(1)<1
$$

for each $n \geqslant 3$, see Section 3.2. This completes the proof of Theorem 2.1.

We also mention that if $a \in\left(0,\left(n e^{2+2 \sqrt{n}}\right)^{-1}\right]$, then

$$
\Lambda_{n}(a)<\left(a n e^{2+2 \sqrt{n}}\right)^{\frac{1}{n}} \leqslant 1 .
$$

Indeed, by Lemma 3.1 and the identities

$$
c_{1}(1)=\sqrt{e}, \quad c_{2}(1)=\frac{3}{2} \sqrt{e}
$$

we have

$$
c_{n}(a)<a c_{n}(1)<a e^{2+2 \sqrt{n}}, \quad n=1,2, \ldots, \quad 0<a<1,
$$

which for each $\gamma \geqslant 1$ yields

$$
\left|g_{n}(z)\right| \geqslant e^{\frac{a}{2}}-\sum_{k=1}^{n} c_{k}|z|^{k}>1-n \cdot a e^{2+2 \sqrt{n}} \gamma^{n}, \quad|z| \leqslant \gamma .
$$

\section{Proofs of Lemmata}

4.1. Proof of Lemma 3.1. It was shown in [7, Sect. 11.2] that the maximal term in the sum expressing $c_{n}(a)$, see Section 3.1 , has the index

$$
p=\left[\frac{1}{2} \sqrt{(a+1)^{2}+4 a n}-\frac{1}{2}(a+1)\right]
$$

here $[x]$ is the greatest integer not exceeding $x$. Thus,

$$
c_{n}(a) \leqslant n e^{\frac{a}{2}}\left(\begin{array}{c}
n-1 \\
p-1
\end{array}\right) \frac{a^{p}}{p !} \equiv e^{\frac{a}{2}} \frac{n ! p a^{p}}{p !^{2}(n-p) !} .
$$

We note that under the assumptions of the lemma we have

$$
p \geqslant 1, \quad n-p \geqslant 1 \text {. }
$$

Indeed, if $p=0$, then

$$
\sqrt{(a+1)^{2}+4 a n}-(a+1)<2,
$$

and hence $n<1+2 / a$. This contradicts to the inequalities $n \geqslant 3 a \geqslant 3$. Hence, $p \geqslant 1$. An easily checked estimate

$$
p \leqslant \sqrt{a n}-\frac{1}{2}(\sqrt{3}-1)(a+1)<\sqrt{a n}
$$

implies the second relation:

$$
1+p<1+\sqrt{a n} \leqslant \frac{n}{3}+\frac{n}{\sqrt{3}}<n
$$

We then have

$$
p>\frac{1}{2} \sqrt{(a+1)^{2}+4 a n}-\frac{1}{2}(a+1)-1>\sqrt{a n}-\frac{1}{2}(a+3)
$$


and therefore,

$$
\sqrt{a n}<p+\frac{1}{2}(a+3)
$$

Transforming the right hand side in (4.1) by means of the Stirling's formula

$$
n !=\sqrt{2 \pi n}\left(n e^{-1}\right)^{n} \varepsilon_{n}, \quad 1<\varepsilon_{n+1}<\varepsilon_{n}<\exp \frac{1}{12 n},
$$

we arrive at the estimate

$$
\frac{c_{n}(a)}{e^{\frac{a}{2}}}<\frac{\sqrt{n} n^{n} a^{p} e^{p}}{2 \pi p^{2 p} \cdot \sqrt{n-p}(n-p)^{n-p}}=\frac{e^{p}}{2 \pi \sqrt{1-\frac{p}{n}}}\left(\frac{\sqrt{a n}}{p}\right)^{2 p}\left(1+\frac{p}{n-p}\right)^{n-p} .
$$

We note that in view of the inequalities 4.2 , 4.3$), a \leqslant n / 3$, we have

$$
2 \pi \sqrt{1-\frac{p}{n}}>2 \pi \sqrt{1-\frac{1}{\sqrt{3}}}>4, \quad \frac{\sqrt{a n}}{p}<1+\frac{a+3}{2 p} .
$$

By inequalities $(1+x)^{1 / x}<e(x>0)$ and 4.2 this yields

$$
c_{n}(a)<e^{\frac{a}{2}} \cdot \frac{e^{p}}{4} e^{a+3} e^{p}=e^{\frac{3 a}{2}+3-\ln 4} e^{2 p}
$$

where

$$
\begin{aligned}
\frac{3}{2} a+3-\ln 4+2 p & \leqslant \frac{3}{2} a+3-\ln 4+2 \sqrt{a n}-(\sqrt{3}-1)(a+1) \\
& =\left(\frac{5}{2}-\sqrt{3}\right)(a+1)+\left(\frac{3}{2}-\ln 4\right)+2 \sqrt{a n} \\
& <a+1+2 \sqrt{a n}
\end{aligned}
$$

The proof of Lemma 3.1 is complete.

Remark 4.1. Let us justify estimate (2.4). In the circle

$$
|z| \leqslant \exp \left(-\frac{2}{\sqrt{3}}-\frac{1}{3}\right)
$$

we have

$$
\left|\sum_{k=n+1}^{\infty} c_{k}\left(\frac{n}{3}\right) z^{k}\right| \leqslant T_{n}:=\sum_{k=n+1}^{\infty} e^{t_{k}}
$$

where, owing to $k \geqslant n+1>50$ and the previous estimate of the quantities $c_{n}(a)$ for $a=n / 3$,

$$
\begin{aligned}
t_{k} & =\left(\frac{5}{2}-\sqrt{3}\right) \frac{n+3}{3}+\left(\frac{3}{2}-\ln 4\right)+\frac{2 \sqrt{n k}}{\sqrt{3}}-\frac{2 k}{\sqrt{3}}-\frac{k}{3} \\
& \leqslant\left(\frac{5}{2}-\sqrt{3}\right) \frac{2}{3}+\left(\frac{3}{2}-\ln 4\right)-\frac{k}{3}\left(\frac{3}{2}-\sqrt{3}\right) \\
& =(0.62567 \ldots-k \cdot 0.01735 \ldots)-k \cdot 0.06 \\
& <-0.06 k .
\end{aligned}
$$

This gives the needed estimate:

$$
T_{n}<e^{-0.06(n+1)}\left(1-e^{-0.06}\right)^{-1}<e^{-3}\left(1-e^{-0.06}\right)^{-1}=0.8549 \ldots<1 .
$$


4.2. Proof of Lemma 3.2. Since $F(x):=e^{-b x}+e^{-b / x} \rightarrow 1(x \rightarrow+\infty)$, it is sufficient to show that $F^{\prime}(x)>0$ as $x>1$.

We have $F^{\prime}(x)=-b e^{-b x}+b x^{-2} e^{-b / x}$. The inequality $F^{\prime}(x)>0(x>1)$ is equivalent to the inequality $e^{b\left(x-x^{-1}\right)}>x^{2}$ and therefore, to the inequality

$$
G(x):=b\left(x-x^{-1}\right)-2 \ln x>0 .
$$

But the latter is true since $G(1)=0$ and in view of $b \geqslant 1$,

$$
G^{\prime}(x)=b\left(1+x^{-2}\right)-2 x^{-1} \geqslant\left(1-x^{-1}\right)^{2}>0 .
$$

The proof of Lemma 3.2 is complete.

\section{BIBLIOGRAPHY}

1. V.I. Danchenko. Approximation properties of sums of the form $\sum_{k} \lambda_{k} h\left(\lambda_{k} z\right) / /$ Matem. Zamet. 83:5, 643-649 (2008). [Math. Notes. 83:5, 587-593 (2008).]

2. P.V. Chunaev. Interpolation by generalized exponential sums with equal weights // J. Approx. Theory. 254, id 105397 (2020).

3. M.A. Komarov. Rate of approximation of $z f^{\prime}(z)$ by special sums associated with the zeros of the Bessel polynomials // Indag. Math. 31:3, 450-457 (2020).

4. P.V. Chunaev, V.I. Danchenko. Approximation by amplitude and frequency operators // J. Approx. Theory. 207, 1-31 (2016).

5. A.V. Fryantsev. On numerical approximation of differential polynomials // Izv. Saratov Univ. (N. S.). Ser. Math. Mech. Inform. 7:2, 39-43 (2007). (in Russian).

6. G.M. Goluzin. Some estimates for bounded functions // Matem. Sborn. 26:1, 7-18 (1950). (in Russian).

7. I.I. Privalov. Boundary properties of analytic functions. GITTL, Moscow (1950). (in Russian).

Mikhail Anatolievich Komarov,

Vladimir State University,

Gorkii str. 87,

600000, Vladimir, Russia

E-mail: kami9@yandex.ru 